\title{
Analysis of Signal Integrity on High Speed Signal Processing System of Vehicle-Mounted Video
}

\author{
X. Wang \\ College of Communications and Information Engineering \\ Nanjing University of Posts and Telecommunications \\ Nanjing, P.R. China
}

\begin{abstract}
The integrity of vehicle mounted video signal refers to all problems caused by the interconnects which transmitted by high rate. However the work frequency of the vehicle electronics becomes higher and the edge of the signal becomes increasingly steep, a larger number of interconnects can not be used as the ideal transmission line to deal with. It makes the signal integrity problems become more complex and has tremendous impact on the stability of the system. There many factors which cause signal integrity, including the signal reflection and crosstalk. In the process of the research, we combine with the advantages of differential signal, such as strong anti-interference capability, timing position accurately and so on. By the way of end termination on differential signal, we reduce the reflection and crosstalk of the signal and the signal integrity is guaranteed.
\end{abstract} signal

Keywords-signal integrity; reflection; crosstalk; differential

\section{INTRODUCTION}

In recent years, the automobile industry of China develops rapidly. At the same time, the progress of computer microelectronics technology and video technology makes more and more high speed signal processing technology be applied to the vehicle video. But as the car working environment is not ideal, which makes the vehicle equipment have to withstand even worse temperature, vibration and shock than in the general environment. This requires the speed of video process work faster and faster, while the problems of signal integrity become more complex [1]. Signal integrity refers to the signal quality on the signal line. The main problems of it include reflection, ground bounce, ringing, and crosstalk and so on. In the process of research, we introduced the differential signal which was applied more and more extensively recently in the full understanding of the signal integrity problems and many factors of the signal integrity problems. And we conduct end termination on differential signal by combining the advantages of differential signal compared to the previous single end signal transmission. Then we do simulation analysis by using related software. The results show that the signal integrity problems of differential signal are improved significantly.

\section{SIGNAL REFLECTION}

Among the many factors which are causing signal integrity problems, signal reflection always disturbs the designers. Signal reflection is that transmission line characteristic impedance can't match load impedance when signal running on the circuit board of abnormal termination. After the signal

\author{
J.S. Tang \\ College of Science \\ Nanjing University of Posts and Telecommunications \\ Nanjing, P. R. China
}

reaches the receiver, a part of the energy will be reflected back along the transmission line, which makes the distortion of signal waveform. It will form a standing wave for analog signals (sinusoidal) and generate overshoot and undershoot phenomena to digital signal [2]. Now we will analyze the important parameters related to signal reflection as shown in Fig. 1 about ideal transmission line circuit model. The resistance of digital power VS in the diagram is RS, so as to drive the $\mathrm{L}$ transmission line. The characteristic impedance of it is Z. Load impedance is ZL. In the ideal situation (critical damping), when $\mathrm{RS}=\mathrm{Z} 0=\mathrm{ZL}$, the impedance of transmission line is continuous. Half of the energy consumed in the source resistance RS, the other half is consumed in the load resistance ZL (transmission line does not have DC loss), so it won't have any reflection. If the load impedance is less than the characteristic impedance of the transmission line, the source end supplies can't meet the energy which load try to consume. Then it will inform the source to provide more energy through the reflection. This is called over-damping. If the characteristic impedance of transmission line is less than the load impedance, the load end can not absorb all the energy fully. Then the remaining energy will be reflected back to the source end. This is called under-damping. It will cause back propagation waveforms whether it is overdamping or under- damping. And it will form a standing wave on transmission lines in some cases.

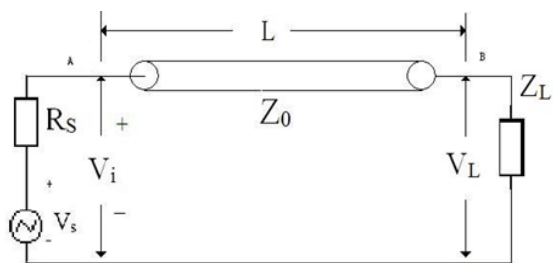

FIGURE I: IDEAL TRANSMISSION LINE MODEL.

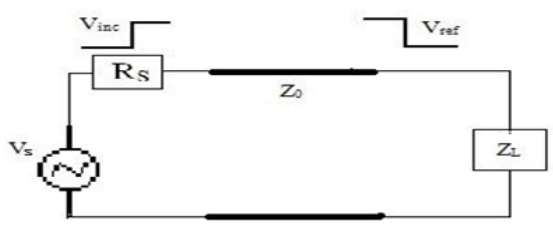

FIGURE II: NON-IDEAL TRANSMISSION LINE MODEL.

By above Fig.2, we know that Reflection coefficient $\mathrm{P}$ is the important index to measure the amount of signal reflection and it is defined as [3]: 


$$
P=\frac{V_{r e f}}{V_{\text {inc }}}=\frac{Z_{L}-Z_{0}}{Z_{L}+Z_{0}}
$$

From the above formula we can see:(1)when $Z L=Z 0$, Vref $=0$,the impedance on both sides of the boundary surface is equal, this case will not have any reflection an we call it critical damping; ( 2 ) when $\mathrm{ZL}<\mathrm{ZO}$, Vref $<0$, Reflection coefficient is negative, this kind of situation is called overdamping, in extreme cases which short circuit will happen at the end of a transmission line and the reflection coefficient $\mathrm{P}=-$ 1 at the same time; ( 3 ) when $Z L>Z 0$, Vref $>0$, Reflection coefficient is upright, this kind of situation is called underdamped, in extreme cases which open circuit will happen at the end of a transmission line and the reflection coefficient $\mathrm{P}=1$ at the same time. Assume that the surface characteristic impedance of the transmission line is $50 \Omega$ and the load resistance is $100 \Omega$,Then the reflection coefficient $P=\frac{100-50}{100+50}=\frac{1}{3}$, so there will be one third signal is reflected back to the source side. If the size of the transmission voltage is 3.6 $\mathrm{V}$, then the reflected signal amplitude of the size is $1.2 \mathrm{~V}$.

Based on above introduction, we can see that reflection is very important for signal integrity, Therefore aimed at reflection we can come to the general principles of designing for high speed signal PCB [4]: Using controlled impedance of interconnect, go line should be as short as possible; Try to reduce the number of via high speed signal; High speed signal should be line with the source and the terminal impedance matching and be Used to eliminate the influence of reflection.

\section{SIGNAL CROSSTALK}

Above, this paper introduces one of the main factors which cause the signal integrity problems; another big factor is the indispensable which is signal crosstalk. Crosstalk is refers to the cable, wires, components and other electronic components which are susceptible to electromagnetic interference and appears the unwanted electromagnetic coupling. Signal crosstalk is that when the signal is transmitted on a transmission line, because of the influence of mutual inductance and mutual capacity, which can produce voltage noise interference and damage to the adjacent transmission line. The interference of a transmission line is too large can cause false triggering of the circuit. As shown in Fig.3, Assuming that located at point A driver is called interference sources, then the receiver at point $\mathrm{D}$ is known as interference of objects, the line network between A and B is called interference sources network, the line network between $\mathrm{C}$ and D network object is called is called interference object network. When the interference sources state changes, it will produce a pulse on the interference objects, In the high speed system, this kind of phenomenon is widespread.

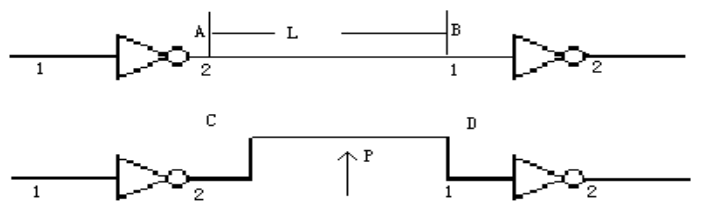

FIGURE III: INTERFERENCE SOURCES AND OBJECTS IN THE CROSSTALK.
For signal integrity, crosstalk will change the performance of the single transmission line such as the characteristics of the transmission line impedance and transmission speed. At the same time, crosstalk noise will induced to other transmission lines which will lead to a decline in the performance of the system. Increase the establishment time of signal, reduce the length of coupling between the transmission line, Increasing the spacing of coupling line and signal lines are placed closely to the formation as far as possible will reduce the crosstalk between signal lines [4].And high speed signal, which In the case of satisfaction, joining termination matching can reduce or eliminate the reflection, which can reduce the crosstalk.

\section{DIFFERENTIAL SIGNAL}

As the signal integrity problems was introduced and the main cause of signal integrity problems is also been introduced, then let us understand the differential signal in this paper. When the driver is driving signal on a transmission line, signal lines and return path will produce a signal voltage which is called single side transmission signal. If the drive of two lines drives a difference, in addition to their single ended signal, there will be a voltage difference between signals which is called differential signal. The transmission is shown in Fig.4, we can see from it that V1 is signal voltage of positive phase transmission line and V2 is signal voltage of reverse phase transmission line. Differential amplifier is to detect the voltage difference between the two voltages, and recover the difference signal [5]: $V_{\text {diff }}=V_{1}-V_{2}$; In the formula, Vdiff is represented as differential signal. Except that the differential signal is used to deliver these carry information, there are common mode signal in the circuit the common mode signal is represented as the average voltage of two transmission lines, it is defined as: $V_{\text {comm }}=\frac{1}{2}\left(V_{1}+V_{2}\right)$.

In this formula, Vcomm is represented as common mode voltage. Making a difference needs only two transmission lines, and the simple single side transmission line can be used to transport. In theory, any two transmission lines can make a difference. Using the difference signal of the difference to transmit compared with the transmission of single ended signal has the following advantages [6]:

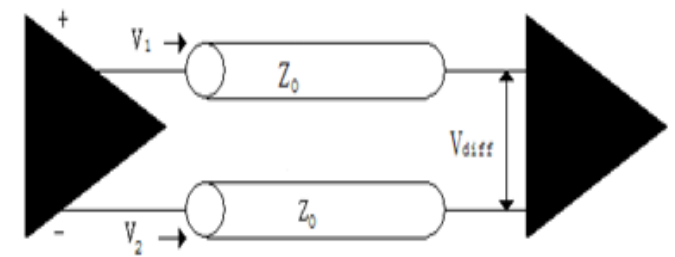

FIGURE IV: STRUCTURE OF DIFFERENTIAL TRANSMISSION.

(1)Compared with the single ended signal, output driver in totally $\mathrm{dI} / \mathrm{dt}$ will be greatly reduced, which can reduce the orbital collapsed and the potential electromagnetic interference;

(2)The differential amplifier of receiver has higher gain than the single-ended amplifier;

(3)The differential signal have its own circuit encapsulation through the connector or been encapsulated, so the interference of switch noise will be less; 
(4)Using cheap twisted pair can implement the differential signal transmission for a long distance.

Differential impedance is the differential signal of the ratio of voltage and current.If a difference signal transmit to the receiving terminal by the differential, the terminal differential impedance will be very big, which can make the difference signal to reflect back to the source side. The multiple reflections will produce noise, and it will influence the quality of the signal. As shown in Fig.5 we get simulation of differential signal which appeared in the end through using the Agilent's ADS to make simulation.(Regardless of the coupling between the difference).

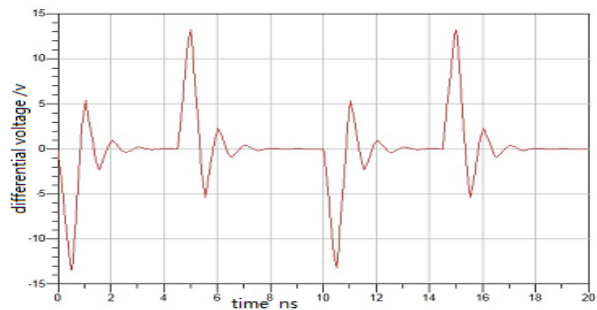

FIGURE V: THE RECEIVED SIGNAL BEFORE MATCHING.

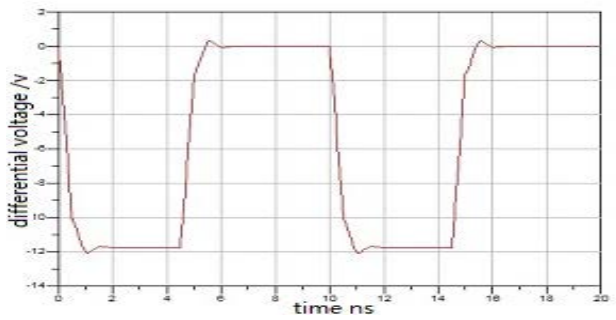

FIGURE VI: THE RECEIVED SIGNAL AFTER MATCHING.

As we can see from the above, the differential signal has distortion during transmission which cannot ensure signal integrity. The main reason is that the difference signal between the driver of a low impedance and high impedance signal terminal bounced many times, which produce the reflection signals and influence the signal integrity. This paper puts forward a method of eliminating reflection is that jumper a termination resistor at the end of the two lines which can be used to match the differential impedance. And this resistance value must be a single-ended signal characteristic impedance of 2 times [7].As shown in Fig. 6, using the differential impedance to match the difference of terminal and then establishing the equivalent circuit model.

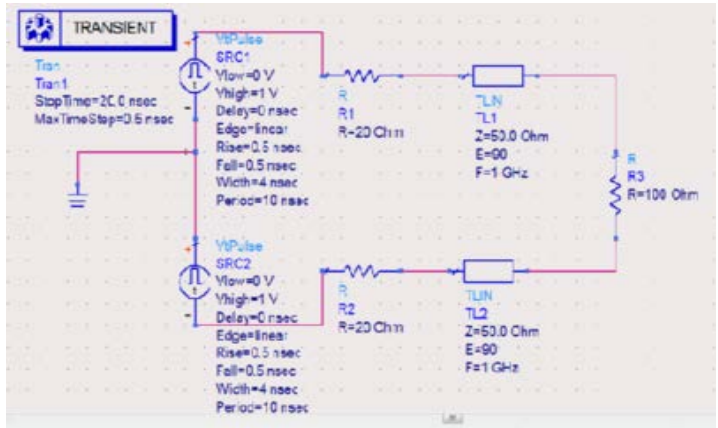

FIGURE VII: THE CIRCUIT MODE OF THE DIFFERENCE.
Then using the Agilent's ADS to simulate, we can get the differential signal which is received distally as shown in Fig.7.From Fig.5 and Fig.7 contrast to be seen that using differential signal to transmit and jumping a termination resistor to match difference impedance at the end of the two lines can eliminate the reflection of the signal so that to reduce the crosstalk signal. Making a difference signal's pushing up, overshoot and ringing getting obvious improvement which is shown in Fig.8, which ensuring the signal integrity.

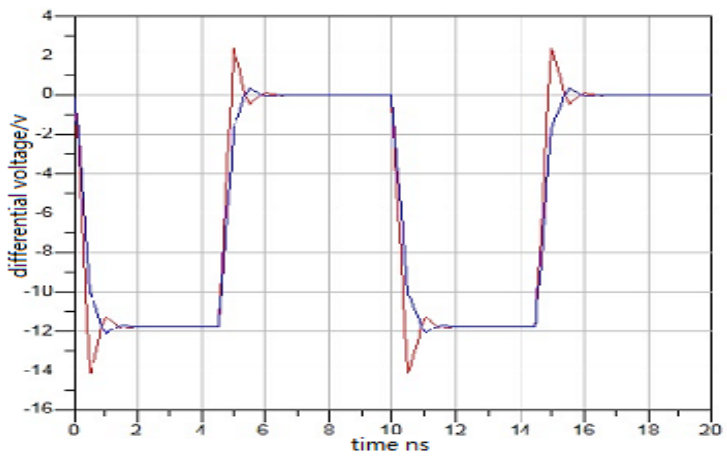

FIGURE VIII: THE CONTRAST FIGURE.

\section{$\mathrm{V} \quad$ CONCLUSIONS}

This paper introduced knowledge related to the on-board video signal integrity, combined with differential signal's characteristics for propagation, using the termination resistor in the end to match the differential impedance so as to eliminate the reflection which can ensure signal integrity. But my level is limited; just consider difference impedance with no coupling. There are two factors which can make the problem complicated, one is the coupling effect between the two lines, the other is the role of the common mode signal and its generation and control [8].So in the later work, I will make deep research on these problems.

\section{REFERENCES}

[1] Stephen H Hall, Garret W Hall. High-Speed Digit System Design[M].Wiley \& Sons ,INC, 2002

[2] Howard W. Johnson, M. Graham "High-Speed Digital Design, A Handbook of Black Magic", Prentice Hall PTR, Englewood Cliffs, New Jersey.

[3] Eric Bogatin, Signal Integrity-Simplified[M]Prentice Hall PTR,2003

[4] Eric Bogatin. Twisted crosstalk [J]. Newsletter v12-01,2009-9-1,2-3

[5] E. Bogatin, Signal integrity: simplified, Beijing: Publishing house of electronics industry, 2005.

[6] B. Yu, The signal integrity design for high-speed electronic circuit, Beijing: Tsinghua University Press, 2004

[7] LI Guang-hui, ZHUANG Yi-qi, ZENG Zhi-bin: Design of a Video Process System Based on Analysis of Signal Integrity. Chinese Journal of Electronic Devices, Aug, 2007?30(4).

[8] F. Jun, Y. Xiaoning, J. Kim, B. Archambeault and A. Orlandi, "Signal Integrity Design for High-Speed Digital Circuits: Progress and Directions,” IEEE Tran. EMC, Vol. 52, No. 2, May 2010, pp. 392-400. 